\title{
-NOTES-
}

\section{VARIATIONAL SOLUTIONS OF THE THOMAS-FERMI EQUATION*}

BY N. ANDERSON AND A. M. ARTHURS (University of York)

Abstract. Variational solutions of the Thomas-Fermi equation are examined in the context of complementary extremum principles. A new one-parameter trial function is found to provide an accurate representation of the solution.

1. Introduction. The Thomas-Fermi screening function $\phi(x)$ for neutral atoms with spherical symmetry satisfies the nonlinear second-order differential equation (see [6])

$$
d^{2} \phi / d x^{2}=\phi^{3 / 2} / x^{1 / 2}, \quad 0 \leq x<\infty,
$$

subject to the boundary conditions

$$
\phi(0)=1 ; \quad \phi \rightarrow 0, \quad x \phi^{\prime} \rightarrow 0 \quad \text { as } \quad x \rightarrow \infty .
$$

Near $x=0$ the function $\phi(x)$ can be expanded as

$$
\phi(x)=1+c x+\frac{4}{3} x^{3 / 2}+\frac{2}{5} c x^{5 / 2}+\frac{1}{3} x^{3}+\cdots,
$$

where $c$ is the unspecified value of $\phi^{\prime}(0)$, while for large $x$ the solution behaves like

$$
\phi(x) \sim 144 / x^{3} \text { as } x \rightarrow \infty .
$$

An analytical solution of the problem in (1) and (2) is not known and so recourse must be taken in approximate solutions. Such solutions are either purely numerical $[3,5]$ or variational $[4,7]$. Here we shall be concerned with solutions of the latter kind.

From the theory of complementary variational principles (see [2]) we find that the solution $\phi$ of (1) and (2) is the function which minimizes the integral

$$
J(\Phi)=\int_{0}^{\infty}\left|\frac{1}{2}\left(\Phi^{\prime}\right)^{2}+\frac{2}{5} \frac{\Phi^{5 / 2}}{x^{1 / 2}}\right| d x,
$$

and which maximizes the integral

$$
G(\Psi)=-\int_{0}^{\infty}\left|\frac{1}{2}\left(\Psi^{\prime}\right)^{2}+\frac{3}{5} \frac{\left(x^{1 / 2} \Psi^{\prime \prime}\right)^{5 / 3}}{x^{1 / 2}}\right| d x-\Psi^{\prime}(0) .
$$

The extreme values of $J$ and $G$ agree and we have the global upper and lower bounds

$$
G(\Psi) \leq G(\phi)=J(\phi) \leq J(\Phi) .
$$

* Received July 1, 1980. 
Here the trial function $\Phi$ is subject to conditions (2) while the trial function $\Psi$ is free of essential conditions. The extremum principles in (7) provide a basis for variational approximations to the exact function $\phi$, the difference $J-G$ being a measure of the accuracy of $\Phi$ and $\Psi$.

2. Variational solutions. The trial function

$$
\Phi_{1}=\left\{a e^{-x x}+b e^{-\beta x}\right\}^{2}, \quad a+b=1,
$$

has been proposed by Csavinsky [4] who determined the three free parameters by a method equivalent to minimizing $J\left(\Phi_{1}\right)$, with the results

$$
J=0.6816
$$

at $a=0.7111, \alpha=0.175, \beta=9.5 \alpha$. For the same class of trial functions the optimum value of the complementary integral $G$ is [1]

$$
G=0.6010 \text {. }
$$

An improvement on these results is provided by the one-parameter trial function

$$
\Phi_{2}=\left(1+\gamma x^{1 / 2}\right) e^{-; x^{1 / 2}}
$$

of Roberts [7], who found the minimum value of $J\left(\Phi_{2}\right)$ to be

$$
J=0.6810 \text { at } \gamma=1.905 \text {. }
$$

The optimum value of $G$ for the same class of trial functions is [1]

$$
G=0.6699 \text { at } \gamma=1.750 .
$$

Neither function $\Phi_{1}$ nor $\Phi_{2}$ has the correct behavior for large $x$, while for small $x$, where the function is most significant, $\Phi_{1}$ has an expansion involving only integral powers of $x$ and cannot look like (3), and $\Phi_{2}$ has the expansion

$$
\Phi_{2}=1-\frac{1}{2} \gamma^{2} x+\frac{1}{3} \gamma^{3} x^{3 / 2}-\frac{1}{8} \gamma^{4} x^{2}+\left(\frac{1}{4 !}-\frac{1}{5 !}\right) \gamma^{5} x^{5 / 2}+O\left(x^{3}\right) .
$$

Comparison of (14) with (3) shows that these expressions agree on the absence of a term in $x^{1 / 2}$, but that $\Phi_{2}$ contains a term in $x^{2}$ which is not present in (3). To incorporate this feature of the exact function $\phi$ near $x=0$, and to retain expansion in powers of $x^{1 / 2}$, we shall therefore modify $\Phi_{2}$ in (11) in the simplest possible way and introduce the oneparameter class of trial functions

$$
\left.\Phi_{3}=\left(1+\alpha x^{1 / 2}+\frac{1}{4} \alpha^{2} x\right) e^{-\alpha 2} x\right) e^{-\alpha x^{1 / 2}} .
$$

Near $x=0$ such a trial function has the expansion

$$
\Phi_{3}=1-\frac{1}{4} \alpha^{2} x+\frac{1}{12} \alpha^{3} x^{3 / 2}-\frac{1}{5 !} \alpha^{5} x^{5 / 2}+\frac{1}{4 ! 12} \alpha^{6} x^{3}+O\left(x^{7 / 2}\right),
$$

the terms in $x^{1 / 2}$ and $x^{2}$ being absent as required.

With function $\Phi_{3}$ the optimum values of $J$ and $G$ and the variational parameter $\alpha$ are

$$
\begin{aligned}
& J=0.6811 \quad \text { at } \quad \alpha=2.472, \\
& G=0.6803 \quad \text { at } \quad \alpha=2.528
\end{aligned}
$$


In terms of the agreement of these bounds the variational solution $\Phi_{3}$ is the best of the three trial functions. Comparison with the numerical solution [3] shows a discrepancy of less than one percent in the region $0 \leq x \leq 1$ where the function drops from 1 to 0.4 . For $x>1, \Phi_{3}$ falls off more rapidly than the numerical solution, but this behavior is to be expected through the built-in exponential decrease in the trial function.

\section{REFERENCES}

[1] N. Anderson, A. M. Arthurs and P. D. Robinson, Nuovo Cimento 57B, 523 (1968)

[2] A. M. Arthurs, Complementary variational principles, Clarendon Press, Oxford, second edition, 1980

[3] V. Bush and S. H. Caldwell, Phys. Rev. 38, 1898 (1931)

[4] P. Csavinszky, Phys. Rev. 166, 53 (1968)

[5] S. Kobayashi, T. Matsukuma, S. Nagai and K. Umeda, J. Phys. Soc. Japan 10, 759 (1955)

[6] L. D. Landau and E. M. Lifshitz, Quantum mechanics, Pergamon Press, Oxford, 1958

[7] R. E. Roberts, Phys. Rev. 170, 8 (1968) 